\title{
Prediction of Parking Availability by Sensor-Enabled Car Parking Slots
}

\author{
Manisha Kulkarni \\ Research Scholar \\ N.B.Navale College of Engineering, Kegaon, \\ Solapur
}

\begin{abstract}
In order to large numbers of vehicles, mainly large numbers of car owners, there are need smart parking system . which system gives the information to car owner or drivers before arrival destination about free parking slot on his /her mobile . proposed system is not only gives the real time information but also gives the prediction about the parking free space. From this technique user can check availability of parking slot for today or tomorrow.
\end{abstract}

This system solve all the problem for searching free space or parking slot, it is big challenge for car drivers to found free space. Because of that air pollution, sound pollution and traffic congestion are created. That's why using of this system user will save our time, money and fuel.

\section{General Terms}

Your general terms must be any term which can be used for general classification of the submitted material such as Pattern Recognition, Security, Algorithms et. al.

\section{Keywords}

Smart cities, smart parking system, internet of things (IOT), KNN, IR sensors, microcontroller, ATmega32., prediction .

\section{INTRODUCTION}

From this paper discussing about the smart parking system in smart cities. peoples are living developed country but they can face some technological problem . But to solve this problem again technology is come to our help. people need smart cities and smart parking system.

A study is state that in France at2006 70 million hours were spent in year for searching a parking slot [2]. Other studies tell that around about $70 \%$ of the world population will start living incites and nearby region by $2050[1]$. One study revels that $30 \%$ of congested traffic in cities is contributed by cars that are searching for parking slots. This system provide system information about available parking space in particular geographic area and process gives real time information to place vehicles at available slot. This system involves low cost sensor, microcontroller, android mobile that allows people to reserve parking in advance and gives very accurate prediction about free parking space availability.

Proposed System is divided in to two sections

Section 1: which is consisting of hardware module.

Section 2: it includes prediction technique using KNN algorithm.

\section{LITREATURE SURVEY}

Author [1] is described prediction technique for parking availability by using simple database. He took data from two major cities. After analyzing mechanism, he decided to

\author{
Shashikant S. Hippargi \\ Professor \\ N.B.Navale College of Engineering, Kegaon \\ Solapur hippargi.shashikant20@gmail.com
}

consider $3 \mathrm{~K}$ parameter which is input data of prediction mechanism then he again uses 3 more non parametric algorithms. These are support vector machines, regression trees, and neural networks.

Author [1] introduced about two new models. One is real time reservation (RTR). Other is share time reservation (STR). In this work RTR could be used by dynamically resource allocation from this drives got parking slot before arriving his / her destination. STR model could be used by allocating static resources based on time scheduling, from this model driver can be choose preferred resources with respect to time frame which will reference in future.

HANIF Et al [4] created systems which used SMS (short messaging services) reservation system. This system includes get access control, remote terminal unit (micro RTU) and microcontroller. Also used pressure sensor for finding occupancy.

Many other technique or system are deployed which giving the availability of parking slots and some are giving reservation facility and some system gives prediction about free parking spaces but those system not able to solve all problem at a time. So proposed gives the real time information about parking availability on your android mobile and also gives prediction for same.

\section{PROPOSED SYSTEM}

This system gives best results over problem of searching free parking space.This system controls traffic congestion, save time and fuel and also avoid air as well as sound pollution.

Proposed system are divided into two sections one gives real time information on android app and other gives prediction about parking slot availability to user.

So paper will be discuss these two sections as below.

\subsection{Section A : Hardware Module}

This system considering some elements like microcontroller ATmega32A,pc,IR sensor, android app, Wi-Fi.

In this work IR sensors have used for receiving data and transmit data to microcontroller and detect and also update status of parking space. It gives information about parking slots are full or empty to system. For this work ATmega32 microcontroller is also used. This is AVR family microcontroller. This is 8 -bit built inADC channel on port A. Android app is developed for provide the real time information to user. User need to install this app on his/her android mobile phone.

Connectivity between system and android application by using Wi-Fi internet, android app link with system by using IP address of the Wi-Fi module. Personal computer is used for keep record of parking status and provide to client. 


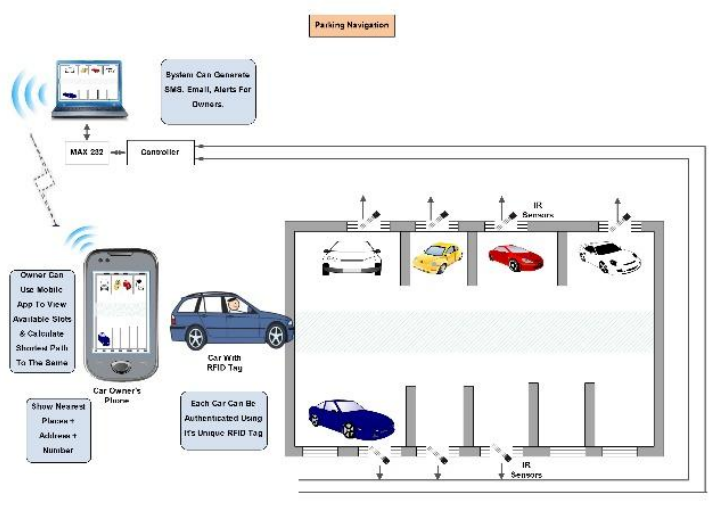

Fig 1 shows the design and implantation of smart parking system

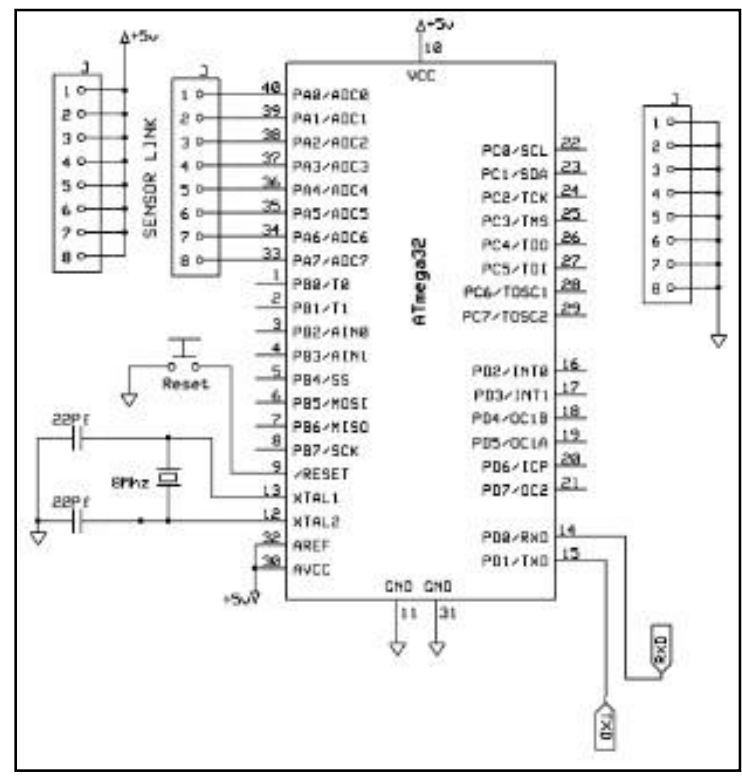

Fig:2 Circuit diagram of hardware module.

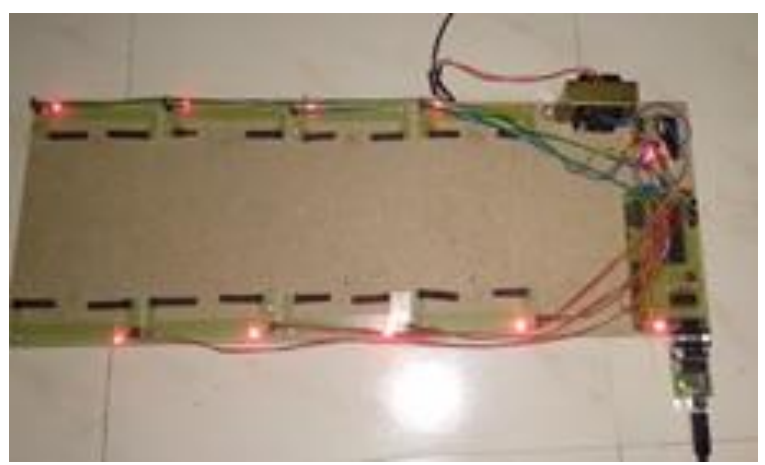

Fig 3 harware module.

\subsection{Section B: Prediction module}

This section involves KNN algorithm, and dataset required for KNN

In this system to predict the availability of parking spaces, an algorithm is selected which gives accuracy and which will work fast. As training dataset is manually created. so supervise learning method in machine learning is used. Supervise learning will provide an algorithm to learn from its training sets. But lots of time supervises learning phases will spend a more time in tasting phase. As this work require fast results so selection of an algorithm which can give result in less time is very important.

KNN can be used based on following parameters

Ease to interpret output - as it can be easy and simple to guess output using KNN compared to other algorithms.

Calculation time - time required for $\mathrm{KNN}$ is very less as training phase is omitted data available in dataset can be used directly for testing phase.

Predictive Power- KNN is most suitable for the prediction.

There is good solution given by using $\mathrm{KNN}$ algorithm for prediction.

KNN did not use not separate different model other than that is stores the entire dataset, by doing this their no learning phase is required. KNN try to make prediction by use of training data set directly.

KNN make prediction for new instances (x) by doing search in complete dataset for the $\mathrm{k}$ most matching instances (considered as neighbors) and by doing summation to the output variable for that $\mathrm{k}$ instances.

\subsection{KNN (K- Nearest Neighbor) Algorithm}

For determining which $\mathrm{k}$ instances in training dataset are most similar to new input distance measure is used most likely Euclidean distance. The value of $\mathrm{k}$ in KNN can be found by algorithm tuning. The computational complexity of KNN increases with size of training set.

KNN works well with small number of input variables (p) but will find difficulties when number of input is very large. To optimize KNN lower dimensionality is preferred KNN can benefits from feature selection that reduces the dimensionality of input feature space.

As selection of $\mathrm{k}$ for prediction there is no such formula. So by using trail and error value of $\mathrm{k}$ will be decided. As in our project system $\mathrm{k}=1$ is considered to find prediction for particular day or time and place

$$
p(i)=\sqrt{\sum_{i=1}^{K}\left(x_{1}-y_{1}\right)^{2}+\left(x_{n}-y_{n}\right)^{2}}
$$

Where $\mathrm{P}(\mathrm{i})$ is predicted values for input $\mathrm{i}$

$\mathrm{X}$ are values taken from datasets which are set of input features.

$\mathrm{Y}$ are value taken from user as input which are set of input features.

by using above formula Euclidean distance is calculated.

By sorting the distance value of nearby neighbor can be calculated with respect to input value.

So topmost value can be considered as predicted value as percentage of availability.

\subsection{Pseudo Code for KNN}

Load data from CSV dataset file Initialize value of $\mathrm{k}$ based on user input value For getting the predicted class it create from 1 to total number of training data point stored in CSV files. 
- Et calculate the distance between test data and each row of training dataset.

- Euclidean distance

- Sort calculated distance in descending order based on distance values

- Get topmost k row from sorted array

- Get most frequent class of that row

- $\quad$ Return the prediction class.

In this work input feature are considered as time slot, month, day, and special occasion as input dataset and same input feature from user to generate prediction value.

Data set is prepared manually for the experimental purpose. Dataset consist of parameter selected for parking of vehicle at particular place. Following parameter are used for training purpose and same parameters. Accuracy parameter is calculated based on occupancy rate which will calculated based on number of parking slot free divided by total number of ,parking slots.

\begin{tabular}{|c|c|c|c|c|c|c|c|}
\hline YEAR & Month & Week & Date & Day & Time_si... & Special ... & Accuracy \\
\hline 2017 & Aug & 1st & 1 & Tuesday & Morning & NO & 17 \\
\hline 2017 & Aug & 1st & 1 & Tuesday & Afternoon & NO & 33 \\
\hline 2017 & Aug & 1st & 1 & Tuesday & Evening & NO & 23 \\
\hline 2017 & Aug & 1st & 2 & Wednes... & Morning & NO & 30 \\
\hline 2017 & Aug & 1st & 2 & Wednes... & Afternoon & NO & 37 \\
\hline 2017 & Aug & 1st & 2 & Wednes... & Evening & NO & 40 \\
\hline 2017 & Aug & 1st & 3 & Thursday & Morning & NO & 7 \\
\hline 2017 & Aug & 1st & 3 & Thursday & Afternoon & NO & 20 \\
\hline 2017 & Aug & 1st & 3 & Thursday & Evening & NO & 33 \\
\hline 2017 & Aug & 1st & 4 & Friday & Morning & NO & 17 \\
\hline 2017 & Aug & 1st & 4 & Friday & Afternoon & NO & 33 \\
\hline 2017 & Aug & 1st & 4 & Friday & Evening & NO & 50 \\
\hline 2017 & Aug & 1st & 5 & Saturday & Morning & NO & 50 \\
\hline 2017 & Aug & 1st & 5 & Saturday & Afternoon & NO & 67 \\
\hline 2017 & Aug & 1st & 5 & Saturday & Evening & NO & 77 \\
\hline 2017 & Aug & 2nd & 6 & Sunday & Morning & NO & 80 \\
\hline 2017 & Aug & 2nd & 6 & Sunday & Afternoon & NO & 67 \\
\hline 2017 & Aug & 2nd & 6 & Sunday & Evening & NO & 40 \\
\hline 2017 & Aug & 2nd & 7 & Monday & Morning & YES & 63 \\
\hline 2017 & Aug & 2nd & 7 & Monday & Afternoon & YES & 73 \\
\hline 2017 & Aug & 2nd & 7 & Monday & Evening & YES & 83 \\
\hline 2017 & Aug & 2nd & 8 & Tuesday & Morning & NO & 53 \\
\hline 2017 & Aug & 2nd & 8 & Tuesday & Afternoon & NO & 33 \\
\hline 2017 & Aug & 2nd & 8 & Tuesday & Evening & NO & 23 \\
\hline 2017 & Aug & 2nd & 9 & Wednes... & Morning & NO & 20 \\
\hline
\end{tabular}

Fig 4 dataset used for KNN

\section{RESULT AND DISCUSSION}

1. Database:

Here MySQL database for experimental purpose. MySQL is open source database management system. It is mostly used for open source client to server.

2. Data processing :

After receiving data, system will perform some comparison with previous data obtained from dataset.

Following figure shows the complete project working

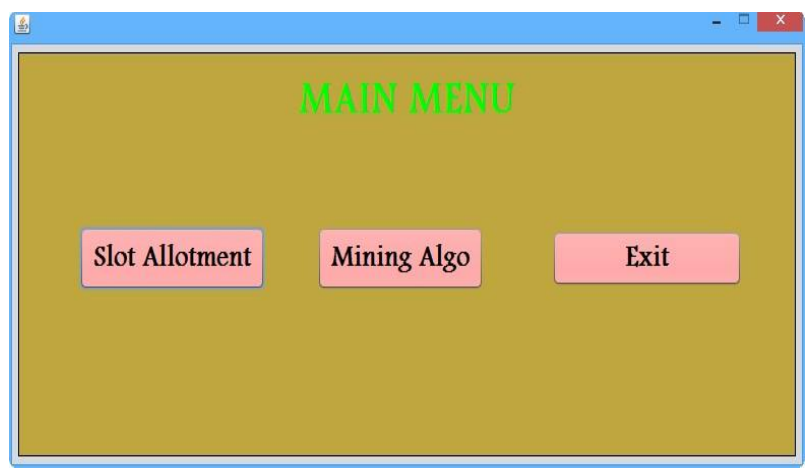

Fig 5. Menu for selecting operation

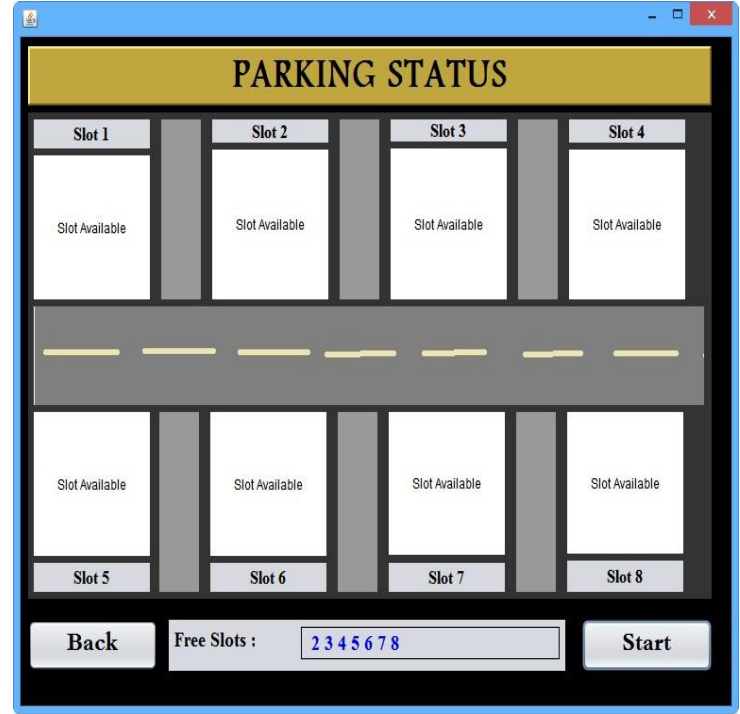

Fig 6. showing All Slot are free

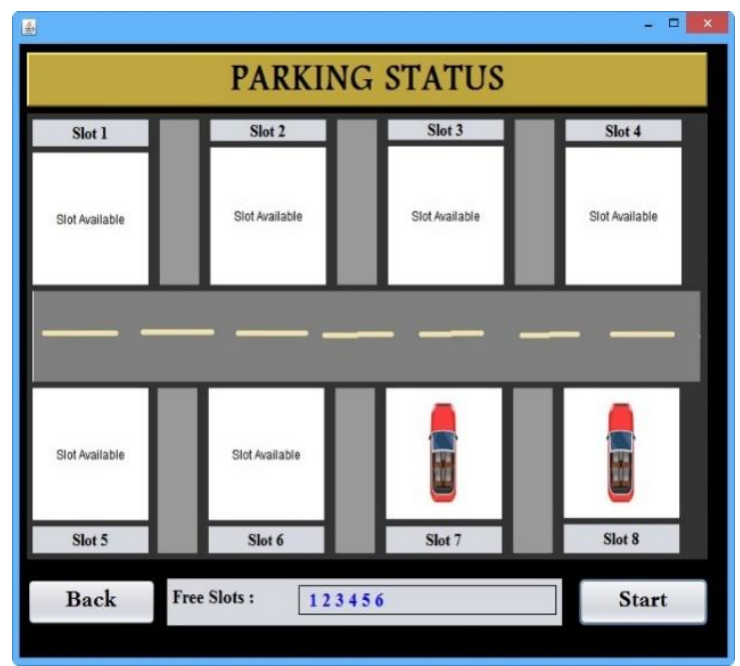

Fig 7. Slot 7 and 8 occupied and remaining is free
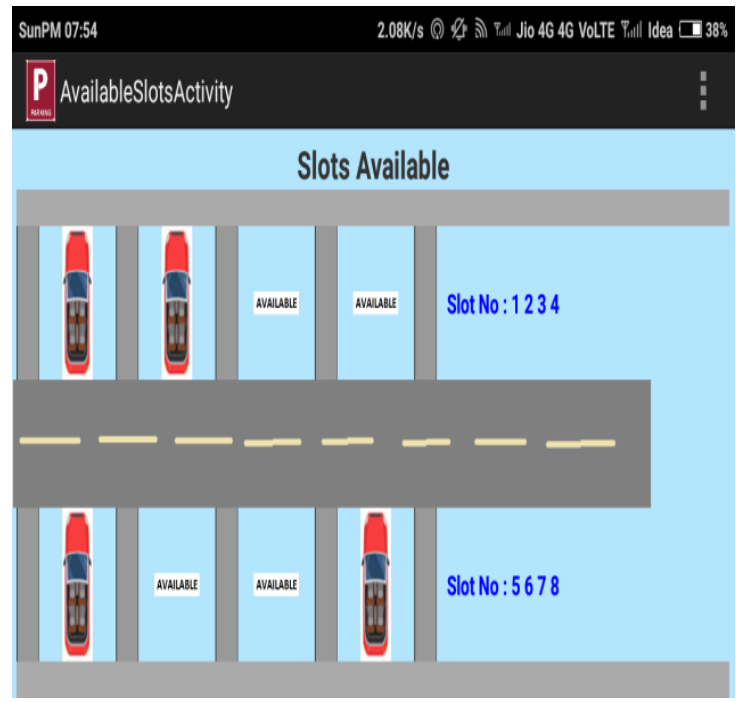

Fig 8 Real time data of parking slot occupied on android mobile app 


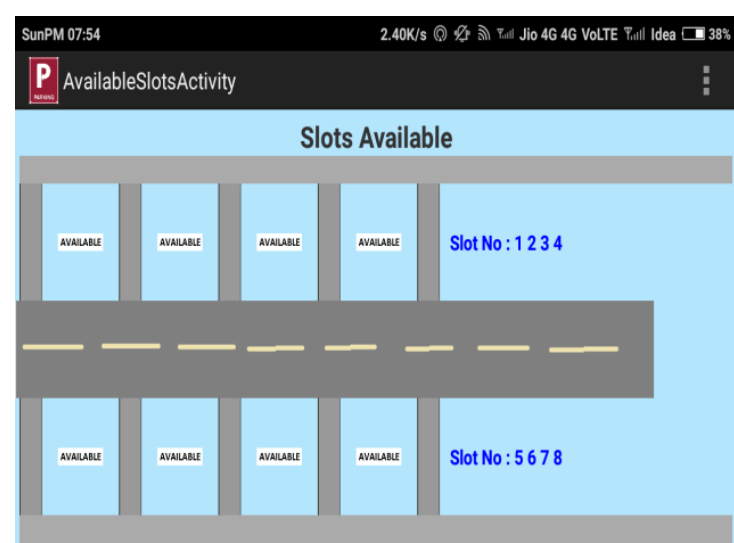

Fig 9 Real time data of free parking slot on android mobile app

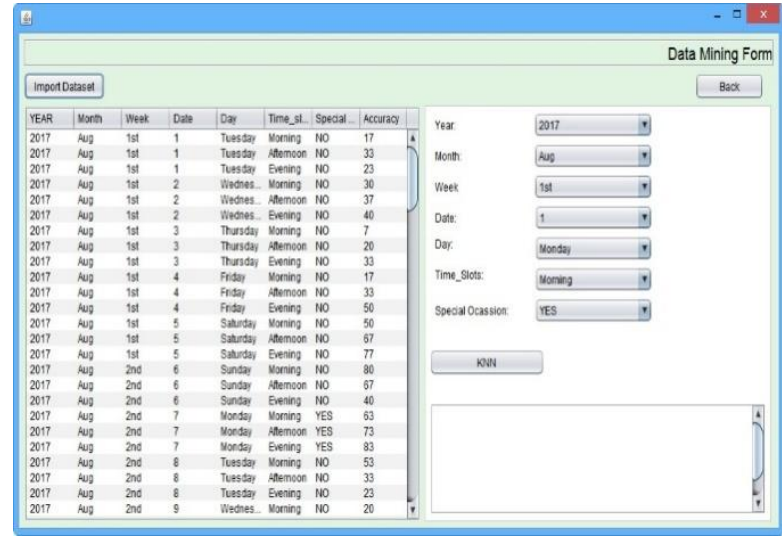

Fig 10. data loaded into prediction module

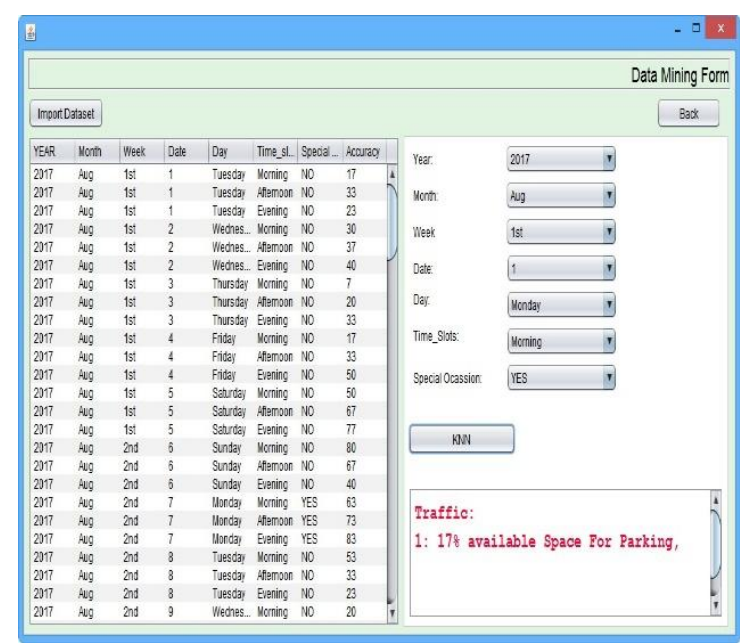

Fig 11. Prediction output 1

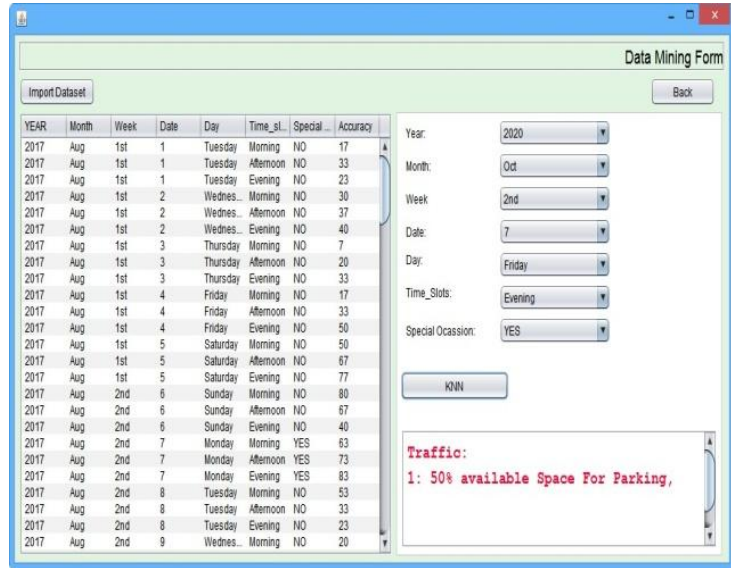

Fig 12. Prediction output 2

\section{CONCLUSION}

This proposed system gives good accurate prediction about parking availability for upcoming dates. Hardware module gives accurate result showing slot which is occupied by demo vehicle which will be again visible on mobile screen using android mobile app. In this work dataset assumed as input for $\mathrm{KNN}$ algorithm for last 3 year, as dataset is larger prediction will be more accurate. In future work can be implement for reservation system by using real time as well predicted values for parking slots

\section{REFERENCES}

[1] Amir, O kotb "iParker - A New smart car parking system based "IEEE 2016. .

[2] K.C Mouskos , "Technical solution to over crowed park and ride "city univ, New York, USA, Tech rep 2007.

[3] Ahmed Yaseen Mjhool "Smart mart parking technique based on internet of thigs , Journal of networks and Telecommunication System 2015

[4] H.D.N Hanif "smart parking reservation system using Short messaging service. 2010.

[5] Yanxu Zheng "parking availability prediction for sensor enabled IEEE 2015.

[6] snehal shinde, Prediction of Parking availability in car parks using sensors and IOT 2016.

[7] advance prediction of parking slot availability with traffic and pollution update for car parks in smart cities. International research journal of engineering and technology 2016.

[8] Microcontroller ATmega32 based automatic vehicle control international journal of scientific and engineering research 2014.

[9] predicting car park occupancy rates in smart cities conference paper June 2017 publisher springer cham Durga Devi TJB ( department of MCA KSRCE)

[10] smart city: IOT based prototype for parking monitoring and management system commanded by mobile App Dr A Subramani ( department of computer science .gov arts college) Dharmapuri India @2017

[11] IoT based smart parking system IEEE A Khanna 2016

[12] A real time parking prediction system for smart cities April 2015 